\title{
Cochrane
Library
}

Cochrane Database of Systematic Reviews

\section{Mobile phone-based interventions for improving adherence to medication prescribed for the primary prevention of cardiovascular disease in adults (Protocol)}

Palmer MJ, Barnard S, Perel P, Free C

Palmer MJ, Barnard S, Perel P, Free C.

Mobile phone-based interventions for improving adherence to medication prescribed for the primary prevention of cardiovascular disease in adults.

Cochrane Database of Systematic Reviews 2017, Issue 5. Art. No.: CD012675.

DOI: 10.1002/14651858.CD012675.

www.cochranelibrary.com

Mobile phone-based interventions for improving adherence to medication prescribed for the primary prevention of cardiovascular disease in adults (Protocol) 
TABLE OF CONTENTS

HEADER . . . . . . . . . . . . . . . . . . . . . . . . . . . . . . . . . . . . . . . 1

ABSTRACT . . . . . . . . . . . . . . . . . . . . . . . . . . . . . . . . . . . . . . . . . . . . .

BACKGROUND . . . . . . . . . . . . . . . . . . . . . . . . . . . . . . . . . . . . .

OBJECTIVES . . . . . . . . . . . . . . . . . . . . . . . . . . . . . . . . . . . . . . . .

METHODS . . . . . . . . . . . . . . . . . . . . . . . . . . . . . . . . . . . . . .

ACKNOWLEDGEMENTS . . . . . . . . . . . . . . . . . . . . . . . . . . . . . . . . . . .

REFERENCES . . . . . . . . . . . . . . . . . . . . . . . . . . . . . . . . . . . . . . 6

APPENDICES . . . . . . . . . . . . . . . . . . . . . . . . . . . . . . . . . . . . . . 7

CONTRIBUTIONS OF AUTHORS . . . . . . . . . . . . . . . . . . . . . . . . . . . . . . . . . . . . . .

DECLARATIONS OF INTEREST . . . . . . . . . . . . . . . . . . . . . . . . . . . . . . . . . . . 9

SOURCES OF SUPPORT . . . . . . . . . . . . . . . . . . . . . . . . . . . . . . . . . . .

Mobile phone-based interventions for improving adherence to medication prescribed for the primary prevention of cardiovascular 


\title{
Mobile phone-based interventions for improving adherence to medication prescribed for the primary prevention of cardiovascular disease in adults
}

\author{
Melissa J Palmer ${ }^{1}$, Sharmani Barnard ${ }^{2}$, Pablo Perel $^{3}$, Caroline Free ${ }^{4}$ \\ ${ }^{1}$ Department of Population Health, London School of Hygiene and Tropical Medicine, London, UK. ${ }^{2}$ Centre for Global Health, \\ Kings College London, London, UK. ${ }^{3}$ Department of Population Health, London School of Hygiene \& Tropical Medicine, London, \\ UK. ${ }^{4}$ Clinical Trials Unit, Department of Population Health, London School of Hygiene \& Tropical Medicine, London, UK
}

Contact address: Melissa J Palmer, Department of Population Health, London School of Hygiene and Tropical Medicine, London, UK. Melissa.palmer@lshtm.ac.uk.

Editorial group: Cochrane Heart Group.

Publication status and date: New, published in Issue 5, 2017.

Citation: Palmer MJ, Barnard S, Perel P, Free C. Mobile phone-based interventions for improving adherence to medication prescribed for the primary prevention of cardiovascular disease in adults. Cochrane Database of Systematic Reviews 2017, Issue 5. Art. No.: CD012675. DOI: 10.1002/14651858.CD012675.

Copyright (C) 2017 The Cochrane Collaboration. Published by John Wiley \& Sons, Ltd.

\begin{abstract}
A B S T R A C T
This is a protocol for a Cochrane Review (Intervention). The objectives are as follows:

To establish the effectiveness of interventions delivered by mobile phone to improve adherence to medication prescribed for the primary prevention of cardiovascular disease in adults.
\end{abstract}

\section{B A C K G R O U N D}

\section{Description of the condition}

Cardiovascular disease (CVD) is a major cause of disability and mortality throughout the world, and contributes substantially to the escalating costs of health care (WHO 2011). An estimated 17.7 million people died from CVDs in 2015, accounting for $31 \%$ of all global deaths (WHO 2016). However, premature fatal and non-fatal CVD is considered to be largely preventable through the control of risk factors (WHO 2011).

Primary prevention of CVD refers to actions taken to reduce the incidence of clinical events due to coronary heart disease (CHD), cerebrovascular disease (CeVD) and peripheral vascular disease, among people with risk factors who have not yet developed clinically manifest CVD (WHO 2007). Primary prevention of CVD consists of lifestyle modifications (e.g. smoking cessation, increasing physical activity) and drug therapy (Piepoli 2016).

Lipid-lowering and anti-hypertensive drug therapies for primary prevention are cost-effective in reducing CVD morbidity and mortality among high-risk people and are recommended by international guidelines (Piepoli 2016; WHO 2007). Recommendations relating to the use of antiplatelet drugs for primary prevention vary. The European Society of Cardiology (ESC) states that aspirin cannot be recommended in primary prevention due to its increased risk of major bleeding (Piepoli 2016); however, the U.S. Preventive Services Task Force (USPSTF) recommends the use of aspirin when the 10-year risk of CVD events reaches such a level that the benefits of aspirin, in terms of CVD events prevented, 
outweigh the potential harm of increased gastrointestinal haemorrhage (USPSTF 2014).

Adherence to long-term medication is less than ideal and results in costs in both health and economic terms (Piepoli 2016). Metaanalyses have estimated rates of adherence to cardiovascular medications ranging from $50 \%$ to $60 \%$ (Chowdhury 2013; Naderi 2012), and there is some evidence that adherence is lower for primary prevention (Naderi 2012).

One study of health records of over 430,000 people in UK general practices found that $47 \%$ of people prescribed statins for primary prevention discontinued treatment (indicated by a greater than 90 day gap between prescriptions), among whom, $72 \%$ who restarted treatment (Vinogradova 2016). One study of Finnish healthcare registers found that $53 \%$ of women prescribed statin therapy for primary prevention were adherent (defined as exceeding $80 \%$ of the prescribed regimen) (Lavikainen 2016). It has been estimated that approximately $9 \%$ of cases of CVDs in the EU could be attributed to poor adherence to vascular medications (Chowdhury 2013). Improving adherence to medications for the primary prevention of CVD would help to maximise the clinical benefits for the wider population (WHO 2003). Therefore, there is considerable scope for increasing adherence to prescribed medicine, and so, reducing morbidity, mortality and healthcare costs.

\section{Description of the intervention}

Mobile phone ownership is almost universal in high-income countries and estimated to have reached over $90 \%$ in low- and middleincome countries (ICT 2016). Given the broad reach of mobile phones and the potential for automation of delivery, interventions delivered by mobile phone are a potentially cost-effective strategy to improve medication adherence. A range of media can be delivered through mobile phones including text messages, picture messages, interactive-voice response, telephone calls and, with increasing ownership of smart phones with Internet capabilities (ICT 2016), mobile applications.

\section{How the intervention might work}

The World Health Organization (WHO) has identified a wide range of factors associated with medication non-adherence (WHO 2003). Mobile phone-based interventions have the potential to target a number of these factors. For example, lack of adherence resulting from poor provider-patient communication and complex/confusing advice or poor recall of information provided in consultations may be addressed through text messages providing short and simply worded snippets of information. Experiences of adverse effects can be targeted through mobile phone-delivered interventions by providing information about medication and facilitating a link to a healthcare professional for people experiencing problems with their medication. Lack of social support has also been linked to poor medication adherence and previous qualitative research found that the receipt of text message-based intervention provided social support (Douglas 2013). Mobile phone-delivered interventions can be designed to target psychological factors such as lack of motivation and low self-efficacy (Free 2016).

Existing interventions targeting adherence to CVD medication have employed mobile technologies to: deliver medication reminders (Park 2014a); encourage self-monitoring of medication intake (Park 2014a); encourage habit formation relating to medication-taking behaviours (Bobrow 2014); provide information (Bobrow 2014; Park 2014a); and facilitate links to healthcare services where required (Bobrow 2014; Piette 2012).

Systematic reviews assessing the effect of mhealth interventions on medication adherence for a range of conditions, including HIV, non-communicable diseases, and prevention of transplant rejection have reported significant improvements (Anglada-Martinez 2015; Park 2014b) and a recent RCT found mobile phone messaging to be effective in improving contraceptive use (Smith 2015). Few adverse effects of mobile phone-based interventions have been reported; potential, but rare, adverse events may include road traffic accidents (Caird 2014).

\section{Why it is important to do this review}

Systematic reviews evaluating the effect of mobile health (mhealth) interventions have reported promising results relating to improved medication adherence (Anglada-Martinez 2015; Park 2014b). One systematic review of interventions delivered by text-messaging targeting medication prescribed for secondary prevention of CVD is currently underway (Adler 2015). However, no systematic review has specifically examined the effect of mobile phone-based interventions on adherence to medications for the primary prevention of CVD. Mobile phone-based interventions are of particular interest given their low-cost and potential for widespread delivery.

\section{O B JE C T I VES}

To establish the effectiveness of interventions delivered by mobile phone to improve adherence to medication prescribed for the primary prevention of cardiovascular disease in adults.

\section{METHOD S}

\section{Criteria for considering studies for this review}


We will include randomised controlled trials (RCTs) of parallel group design that randomise by participant or by cluster. We will not include cross-over trials as this design would be inappropriate for assessing effects on cardiovascular events or mortality, due to the irreversible of these events. We will only include trials with a minimum of one-year follow-up in order that the outcome measures relate to longer-term, sustained medication adherence behaviours and outcomes. We will include studies published as full text and as abstract only, and unpublished data.

\section{Types of participants}

We will include adults (aged 18 years and over) who have been prescribed medication for the primary prevention of CVD. As this review will focus on the primary prevention of CVD, we will only include studies involving participants who have not had a prior CVD event, defined as: a previous myocardial infarction (MI), stroke, revascularisation procedure (coronary artery bypass grafting $(\mathrm{CABG})$ or percutaneous coronary intervention $(\mathrm{PCI})$ ), people with angina, and people with angiographically defined CHD. Where we identify trials that include a subset of eligible participants, we will contact the authors to request data for only those participants of interest. In the event that we are unable to access these data, we will apply a cut-off whereby only trials in which at least $75 \%$ of participants meet the criteria for primary prevention will be included.

\section{Types of interventions}

We will include trials of interventions delivered wholly or partly by mobile phone to improve adherence to cardiovascular medications prescribed for the primary prevention of CVD. We will include interventions targeting adherence to antihypertensive drugs (thiazide-like diuretic, angiotensin-converting enzyme (ACE) inhibitor, calcium channel blocker, beta-blocker); lipid-lowering drugs (statins); and antiplatelet drugs (low-dose aspirin, non-aspirin antiplatelet drugs). We will only include trials targeting adherence to at least one of these medications. We will also include trials of interventions that target medication adherence alongside other lifestyle modifications.

Intervention: any mobile phone-specific delivery mechanism, including short messaging service (SMS), multimedia messaging (MMS), applications (apps) and Interactive Voice Response (IVR). We will include interventions employing a mix of delivery mechanisms of which at least one is mobile phone-based, for example, interventions delivered by mobile phones in combination with traditional methods such as face-to-face communication and links to other types of support (e.g. healthcare support worker, telephone calls, web pages).

Comparator: usual care and active controls where the control group intervention has no component delivered by a mobile phone-specific delivery mechanism.

\section{Types of outcome measures}

\section{Primary outcomes}

- Objective measures of adherence to treatment (low-density lipoprotein (LDL)-cholesterol for the effect of statins; blood pressure for antihypertensive drugs; heart rate for the effect of atenolol; urinary 11-dehydrothromboxane B2 for the antiplatelet effects of aspirin).

- Combined CVD event (fatal or non-fatal events).

- Adverse effects including self-reported road traffic accidents.

\section{Secondary outcomes}

- Indirect measures of adherence to treatment (self-report, pill counts, medication event monitoring systems (MEMS), pharmacy prescription data).

- Fatal cardiovascular events.

- Non-fatal cardiovascular events (CHD, stroke).

- Health-related quality of life assessed using validated instruments (e.g. 36-Item Short Form Health Survey (SF-36), EQ-5D).

- Cognitive outcomes (any measures of: satisfaction with medication, medication-taking self-efficacy, autonomy related to medication, attitudes (e.g. concerns about medicine adverse effects)).

- Costs.

We will also report on the following process measures: extent of intervention received (e.g. number of text messages received, measures of use of allocated mobile application) and acceptability of intervention.

Reporting one of more of the outcomes listed here in the trial will not be an inclusion criterion for the review.

Where outcomes (primary or secondary) are measured at multiple time points, we will extract data for the final point of measurement.

\section{Search methods for identification of studies}

\section{Electronic searches}

We will identify trials through systematic searches of the following bibliographic databases from inception to the date of search:

- Cochrane Central Register of Controlled Trials

(CENTRAL) in the Cochrane Library;

- MEDLINE (Ovid);

- Embase (Ovid);

- CINAHL Plus (EBSCOhost);

- Conference Proceedings Citation Index-Science (CPCI-S) on Web of Science (Thomson Reuters). 
The preliminary search strategy for MEDLINE (Ovid) (Appendix 1) will be adapted for use in the other databases. The Cochrane sensitivity-precision maximising RCT filter (Lefebvre 2011) will be applied to MEDLINE (Ovid) and adaptations of it to the other databases, except CENTRAL.

We will carry out a search of ClinicalTrials.gov (www.ClinicalTrials.gov) and the WHO International Clinical Trials Registry Platform (ICTRP) Search Portal (apps.who.int/trialsearch/) for ongoing or unpublished trials.

We will impose no restriction on language of publication.

We will not perform a separate search for adverse effects of mobile phone-based interventions targeting medication adherence. We will consider adverse effects described in included studies only.

\section{Searching other resources}

We will check the reference lists of all included studies and we will review relevant articles for additional references. We will also examine any relevant retraction statements and errata for included studies.

\section{Data collection and analysis}

\section{Selection of studies}

Two review authors (MP and SB) will independently screen the titles and abstracts of all identified potential studies to decide whether to retrieve the full text (eligible or potentially eligible/unclear studies) or to discard the study. Two review authors (MP and SB) will independently screen the retrieved full texts to identify studies for inclusion and identify and record reasons for exclusion of the ineligible studies in a 'Characteristics of excluded studies' table. We will endeavour to resolve any disagreements though discussion, and if necessary, a third review author (CF or PP) will arbitrate. We will exclude any duplicates. Multiple reports of the same RCT study will be collated into a single entry. We will complete a PRISMA flow diagram.

\section{Data extraction and management}

We will use a standardised, prepiloted form to extract data from the included studies for assessment of study quality and evidence synthesis. We will contact chief investigators for additional information if necessary. We will extract the following information.

- Methods: study design; total duration of study; study setting and date of study.

- Participants: number randomised; number lost to followup/withdrawn; number analysed; mean age; age range; gender; proportion meeting criteria of 'primary prevention'; and inclusion criteria and exclusion criteria.
- Interventions: intervention; comparison; concomitant medications; excluded medications; intervention delivery mechanism (text messages/MMS/mobile application/combined); how intervention was developed; behaviour change technique(s) employed; if intervention was personalised; and frequency and duration of intervention receipt.

- Outcomes: primary and secondary outcomes specified and collected; adverse effects; and time points reported.

- Notes: funding for trial and notable conflicts of interest of trial authors.

Two review authors (MP and SB) will independently extract data and resolve any differences by returning to the original study reports and discussion with a third review author (CF or PP) where necessary. One review author (MP) will transfer data into the Review Manager 5 (RevMan 2014). To ensure that there are no errors in data entry, one review author (SB) will check that the data entered into Review Manager 5 are consistent with those in the data extraction form.

\section{Assessment of risk of bias in included studies}

Two review authors (MP and SB) will independently assess the risk of bias for each study using the criteria detailed in the Cochrane Handbook for Systematic Reviews of Interventions (Higgins 2011). For each of the following domains, we will grade the potential bias as high, low or unclear.

- Random sequence generation.

- Allocation concealment.

- Blinding of participants and personnel.

- Blinding of outcome assessment.

- Incomplete outcome data.

- Selective outcome reporting.

- Other biases.

We will resolve any disagreements by discussion. Where necessary, we will consult a third review author (CF or PP) to arbitrate. We will construct a 'Risk of bias' table including justifications for our judgements. Where information relating to the risk of bias has come from unpublished data or correspondence with an author, we will note this. We will summarise the risk of bias judgements across different studies for each of the domains listed. When considering treatment effects, we will take into account the risk of bias for the studies that contribute to that outcome.

Given the nature of the interventions to be included in this review, it is likely that blinding of participants and personnel will be impossible, therefore, we expect trials to be categorised at high risk of bias on this domain. For the overall study assessment, we will categorise a trial as being at low risk of bias if it was rated as low risk in all the domains listed above (with the exception of blinding of participants and personnel). Trials that have been rated as high or unclear risk of bias on any of the domains (except blinding of participants and personnel) will be categorised as being at high risk of bias. 


\section{Assessment of bias in conducting the systematic review}

We will conduct the review according to this published protocol and report any deviations from it in the 'Differences between protocol and review' section of the systematic review.

\section{Measures of treatment effect}

We will analyse dichotomous outcome data as risk ratios with $95 \%$ confidence intervals. We will analyse continuous outcome data as mean differences with $95 \%$ confidence intervals, or if a continuous outcome has been measured in multiple ways, as a standardised mean difference with $95 \%$ confidence intervals. We will enter data presented as a scale with a consistent direction of effect. We will report any skewed data identified as medians and interquartile ranges.

\section{Unit of analysis issues}

We will include RCTs with parallel design. If we identify cluster RCTs for inclusion, we will analyse the data accounting for clustering using the intracluster coefficient (ICC).

If we identify multi-arm trials for inclusion, where there is more than one relevant intervention arm but only one control arm, the intervention arms will be pooled for a single pair-wise comparison as recommended by the Cochrane Handbook for Systematic Reviews of Interventions (Higgins 2011). We will exclude intervention arms not appropriate for this review.

\section{Dealing with missing data}

We will contact investigators or study sponsors to obtain missing data where possible (e.g. when a study is identified as abstract only). Where this is not possible, and the missing data are considered a potential source of serious bias, we will conduct a sensitivity analysis to explore the impact of including such studies in the overall assessment of results.

\section{Assessment of heterogeneity}

We will use the $\mathrm{I}^{2}$ statistic to measure heterogeneity across the trials for the analysis of each outcome. Where we identify there to be moderate to substantial heterogeneity (an $\mathrm{I}^{2}$ statistic between $30 \%$ and $100 \%$ ), we will report it and examine possible causes according to our prespecified subgroup analyses.

\section{Assessment of reporting biases}

If the results from more the 10 trials can be pooled, we will use a funnel plot to explore possible small-study biases for the primary outcomes.

\section{Data synthesis}

We will carry out meta-analyses only if it considered meaningful to do so (i.e. if the interventions, participants and outcome measures are similar enough for pooling to make sense). In the absence of heterogeneity, we will use fixed-effect models. In the presence of heterogeneity (an $\mathrm{I}^{2}$ statistic in excess of $30 \%$ ), we will provide a narrative overview without pooling data for analysis.

\section{'Summary of findings' table}

Two review authors (MP and SB) will use the five GRADE considerations (study limitations, consistency of effect, imprecision, indirectness and publication bias) to assess the quality of a body of evidence as it relates to the studies that contribute data to the meta-analyses for the following outcomes: objective measures of adherence to treatment, combined CVD event (fatal and non-fatal events), adverse events and cognitive outcomes. We will use methods and recommendations described in Section 8.5 and Chapter 12 of the Cochrane Handbook for Systematic Reviews of Interventions (Higgins 2011) using GRADEpro software. We will justify all decisions to downgrade the quality of studies using footnotes and we will make comments to aid readers' understanding of the review where necessary.

\section{Subgroup analysis and investigation of heterogeneity}

If there are sufficient studies, we will carry out the following subgroup analyses for the primary outcome of adherence to treatment:

- income region (by World Bank income group) (World Bank 2017);

- how text messages were developed (i.e. theory-based, incorporating user views and based on evidence relating to factors influencing behaviour-targeted versus other);

- intervention content (number behaviour change technique employed coded according to the taxonomy developed by Michie and colleagues (Michie 2015));

- delivery mechanisms (i.e. mobile phone messaging only, mobile applications only, combined mobile phone messaging and application, combined application and other).

We will use the formal test for subgroup interactions in Review Manager 5 (RevMan 2014).

\section{Sensitivity analysis}

We plan to carry out the following sensitivity analyses.

- Only including studies with low risk of bias.

\section{Reaching conclusions}

We will base our conclusions only on findings from the quantitative or narrative synthesis of included studies for this review. We 
will avoid making recommendations for practice and our implications for research will suggest priorities for future research and outline what the remaining uncertainties are in the area.

\section{ACKNOWLEDGEMENTS}

The review authors would like to acknowledge assistance provided by staff of the Cochrane Heart Group and the template protocol they made available.

\section{R E F E R E N C E S}

\section{Additional references}

Adler 2015

Adler AJ, Martin N, Mariani J, Tajer CD, Serrano NC, Casas JP, et al. Mobile phone text messaging to improve adherence to cardiovascular disease secondary prevention interventions. Cochrane Database of Systematic Reviews 2015, Issue 8. [DOI: 10.1002/14651858.CD011851]

Anglada-Martinez 2015

Anglada-Martinez H, Riu-Viladoms G, Martin-Conde M, Rovira-Illamola M, Sotoca-Momblona JM, Codina-Jane C. Does mHealth increase adherence to medication? Results of a systematic review. International Journal of Clinical Practice 2015;69(1):9-32.

Bobrow 2014

Bobrow K, Brennan T, Springer D, Levitt NS, Rayner B, Namane M, et al. Efficacy of a text messaging (SMS) based intervention for adults with hypertension: protocol for the StAR (SMS Text-message Adherence suppoRt trial) randomised controlled trial. BMC Public Health 2014;14: 28. [DOI: 10.1186/1471-2458-14-28]

Caird 2014

Caird JK, Johnston KA, Willness CR, Asbridge M, Steel P. A meta-analysis of the effects of texting on driving. Accident Analysis \& Prevention 2014;71:311-8.

Chowdhury 2013

Chowdhury R, Khan H, Heydon E, Shroufi A, Fahimi $S$, Moore C, et al. Adherence to cardiovascular therapy: a meta-analysis of prevalence and clinical consequences. European Heart Journal 2013;34(38):2940-8.

Douglas 2013

Douglas N, Free C. 'Someone batting in my corner': experiences of smoking-cessation support via text message. British Journal of General Practice 2013;63(616):e768-76.

Free 2016

Free C, McCarthy O, French RS, Wellings K, Michie $S$, Roberts I, et al. Can text messages increase safer sex behaviours in young people? Intervention development and pilot randomised controlled trial. Health Technology Assessment (Winchester, England) 2016;20(57):1-82.
Higgins 2011

Higgins JP, Green S, editor(s). Cochrane Handbook for Systematic Reviews of Interventions Version 5.1.0 (updated March 2011). The Cochrane Collaboration, 2011. Available from handbook.cochrane.org.

\section{ICT 2016}

ICT. Key ICT indicators for developed and developing countries and the world, 2016. www.itu.int/en/ITU-D/ Statistics/Pages/facts/default.aspx (accessed 6 October 2016).

\section{Lavikainen 2016}

Lavikainen P, Helin-Salmivaara A, Eerola M, Fang G, Hartikainen J, Huupponen R, et al. Statin adherence and risk of acute cardiovascular events among women: a cohort study accounting for time dependent confounding affected by previous adherence. BMJ Open 2016;6(6):e011306. [DOI: 10.1136/bmjopen-2016-011306]

Lefebvre 2011

Lefebvre C, Manheimer E, Glanville J. Chapter 6: Searching for studies. In: Higgins JP, Green S, editor(s). Cochrane Handbook for Systematic Reviews of Interventions Version 5.1.0 (updated March 2011). The Cochrane Collaboration, 2011. Available from handbook.cochrane.org.

\section{Michie 2015}

Michie S, Wood CE, Johnston M, Abraham C, Francis JJ, Hardeman W. Behaviour change techniques: the development and evaluation of a taxonomic method for reporting and describing behaviour change interventions (a suite of five studies involving consensus methods, randomised controlled trials and analysis of qualitative data). Health Technology Assessment 2015;19(99):1-187.

\section{Naderi 2012}

Naderi SH, Bestwick JP, Wald DS. Adherence to drugs that prevent cardiovascular disease: meta-analysis on 376,162 patients. American Journal of Medicine 2012;125(9):882-7.

Park 2014a

Park LG, Howie-Esquivel J, Chung ML, Dracup K. A text messaging intervention to promote medication adherence for patients with coronary heart disease: a randomized

Mobile phone-based interventions for improving adherence to medication prescribed for the primary prevention of cardiovascular 
controlled trial. Patient Education and Counselling 2014;94 (2):261-8.

\section{Park 2014b}

Park LG, Howie-Esquivel J, Dracup K. A quantitative systematic review of the efficacy of mobile phone interventions to improve medication adherence. Journal of Advanced Nursing 2014;70(9):1932-53.

\section{Piepoli 2016}

Piepoli MF, Hoes AW, Agewall S, Albus C, Brotons C, Catapano AL, et al. 2016 European Guidelines on cardiovascular disease prevention in clinical practice The Sixth Joint Task Force of the European Society of Cardiology and Other Societies on Cardiovascular Disease Prevention in Clinical Practice (constituted by representatives of 10 societies and by invited experts) developed with the special contribution of the European Association for Cardiovascular Prevention \& Rehabilitation (EACPR). European Journal of Preventive Cardiology 2016;23(11):NP1-NP96.

\section{Piette 2012}

Piette JD, Datwani H, Gaudioso S, Foster SM, Westphal J, Perry W, et al. Hypertension management using mobile technology and home blood pressure monitoring: results of a randomized trial in two low/middle-income countries. Telemedicine and e-Health 2012;18(8):613-20.

RevMan 2014 [Computer program] Nordic Cochrane Centre, The Cochrane Collaboration. Review Manager (RevMan). Version 5.3. Copenhagen: Nordic Cochrane Centre, The Cochrane Collaboration, 2014.

\section{Smith 2015}

Smith C, Ngo TD, Gold J, Edwards P, Vannak U, Sokhey $\mathrm{L}$, et al. Effect of a mobile phone-based intervention on post-abortion contraception: a randomized controlled trial in Cambodia. Bulletin of the World Health Organization 2015;93(12):842-50A.

\section{USPSTF 2014}

U.S. Preventive Services Task Force. The guide to clinical preventive services: recommendations of the U.S. Preventive Services Task Force, 2014. www.uspreventiveservicestaskforce.org/Page/Name/toolsand-resources-for-better-preventive-care (accessed 11 April 2017).

\section{Vinogradova 2016}

Vinogradova Y, Coupland C, Brindle P, Hippisley-Cox J. Discontinuation and restarting in patients on statin treatment: prospective open cohort study using a primary care database. BMJ 2016;353:i3305. [DOI: https:// doi.org/10.1136/bmj.i3305]

\section{WHO 2003}

World Health Organization. Adherence to long-term therapies: evidence for action, 2003. www.who.int/chp/ knowledge/publications/adherence_report/en/ (accessed 15 February 2017).

\section{WHO 2007}

World Health Organization. Prevention of cardiovascular disease: guidelines for assessment and management of cardiovascular risk, 2007. www.who.int/cardiovascular_ diseases/publications/Prevention_of_Cardiovascular_ Disease/en/ (accessed 15 February 2017).

\section{WHO 2011}

World Health Organization. Global Atlas on Cardiovascular Disease Prevention and Control, 2011. www.who.int/ cardiovascular_diseases/publications/atlas_cvd/en/ (accessed 15 February 2017).

\section{WHO 2016}

World Health Organization. Cardiovascular diseases (CVDs) Fact Sheet No317, 2016. www.who.int/ mediacentre/factsheets/fs317/en/ (accessed 24 May 2017).

\section{World Bank 2017}

World Bank. World Development Indicators 2017. Washington, DC: World Bank. License: Creative Commons Attribution CC BY 3.0 IGO. https:// openknowledge.worldbank.org/handle/10986/26447 (accessed 30 May 2017) 2017.

* Indicates the major publication for the study 


\section{A P P E N D I C E S}

\section{Appendix I. Preliminary MEDLINE (Ovid) search strategy}

1 exp Cell Phones/

2 ((cell* or mobile) adj (phone* or telephon*)).tw.

3 (cellphone* or mobiles or smartphone*).tw.

4 ((mobile or handheld or hand-held or cell* or phone*) adj2 (device* or technolog* or app* or health*)).tw.

5 Text Messaging/

6 sms.tw.

7 ((text or short or multimedia or multi-media or mms) adj1 messag*).tw.

8 (texting* or texted or texter*).tw.

9 Telemedicine/

10 (mhealth or m-health or ehealth or e-health or telemedicine* or telehealth or telemonitor*).tw.

11 Reminder Systems/

12 (reminder* adj (text* or system* or messag*)).tw.

131 or 2 or 3 or 4 or 5 or 6 or 7 or 8 or 9 or 10 or 11 or 12

14 exp Cardiovascular Diseases/

15 cardio*.tw.

16 cardia*.tw.

17 heart*.tw.

18 coronary*.tw.

19 angina*.tw.

20 ventric*.tw.

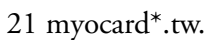

22 pericard*.tw.

23 isch?em*.tw.

24 emboli*.tw.

25 arrhythmi*.tw.

26 thrombo*.tw.

27 atrial fibrillat*.tw.

28 tachycardi*.tw.

29 endocardi*.tw.

30 (sick adj sinus).tw.

31 hypertensi*.tw.

32 exp Hyperlipidemias/

33 hyperlipid*.tw.

34 hyperlip?emia*.tw.

35 hypercholesterol*.tw.

36 hypercholester?emia*.tw.

37 hyperlipoprotein?emia*.tw.

38 hypertriglycerid?emia*.tw.

39 arteriosclerosis.tw.

40 atherosclerosis.tw.

41 exp Cholesterol/

42 cholesterol.tw.

43 Blood Pressure/

44 ((high* or raise* $^{*}$ or elevat* ${ }^{*}$ or heighten* or increas* $^{*}$ adj3 (blood adj2 pressure)).tw.

45 ((high* or raise* $^{*}$ or elevat* ${ }^{*}$ or heighten* ${ }^{*}$ or increas*) adj3 (BP or DBP or SBP)).tw.

46 ((diastolic or systolic or pulse) adj pressure).tw.

47 exp Stroke/

48 (stroke or strokes).tw.

Mobile phone-based interventions for improving adherence to medication prescribed for the primary prevention of cardiovascular 
49 cerebrovasc*. tw.

50 cerebral vascular.tw.

51 apoplexy.tw.

52 ((brain* or cerebral or lacunar) adj2 infarct*).tw.

53 peripheral arter* disease*.tw.

54 aortic*.tw.

55 (arterial adj occlus*).tw.

56 infarct*.tw.

57 multiple risk factor.tw.

5814 or 15 or 16 or 17 or 18 or 19 or 20 or 21 or 22 or 23 or 24 or 25 or 26 or 27 or 28 or 29 or 30 or 31 or 32 or 33 or 34 or 35 or 36 or 37 or 38 or 39 or 40 or 41 or 42 or 43 or 44 or 45 or 46 or 47 or 48 or 49 or 50 or 51 or 52 or 53 or 54 or 55 or 56 or 57

59 randomized controlled trial.pt.

60 controlled clinical trial.pt.

61 randomized.ab.

62 placebo.ab.

63 clinical trials as topic.sh.

64 randomly.ab.

65 trial.ti.

6659 or 60 or 61 or 62 or 63 or 64 or 65

67 exp animals/ not humans.sh.

6866 not 67

6913 and 58 and 68

\section{CONTRIBUTIONSOFAUTHORS}

MP: registered the title with the Cochrane Heart Group and prepared the first draft of this protocol.

SB: contributed to designing and writing the protocol.

PP: contributed to designing and writing the protocol.

CF: conceived the idea for this review, led on designing the protocol and contributed to writing the protocol.

\section{DECLARATIONS OF INTEREST}

MP: none known.

SB: none known.

PP: is the principal investigator for a study developing and piloting an mHealth intervention to increase adherence for cardiovascular secondary prevention interventions.

CF: we have developed an intervention delivered by text message designed to increase adherence to medication to prevent cardiovascular disease. We are likely to apply for funding for a RCT to evaluate it effects. 


\section{SOURCES OF SUPPORT}

\section{Internal sources}

- No sources of support supplied

\section{External sources}

- The Cochrane Heart Group US Satellite is supported by intramural support from the Northwestern University Feinberg School of Medicine and the Northwestern University Clinical and Translational Science (NUCATS) Institute (UL1TR000150), USA.

- This project was supported by the National Institute for Health Research (NIHR), via Cochrane Infrastructure funding to the Heart Group. The views and opinions expressed therein are those of the authors and do not necessarily reflect those of the Systematic Reviews Programme, NIHR, National Health Service or the Department of Health, UK. 